Düşük taper açısına sahip güncel NiTi döner aletlerin döngüsel

yorgunluk dirençlerinin kıyaslanması

\section{Comparison of cyclic fatigue resistance of novel NiTi rotary instruments with low taper angle}

\author{
Dr. Öğr. Üyesi Ahmet Demirhan Uygun \\ Afyonkarahisar Sağlık Bilimleri Üniversitesi, Diş He- \\ kimliği Fakültesi, Endodonti A.D., Afyonkarahisar \\ Orcid ID: 0000-0001-5704-183X
}

\section{Dr. Öğr. Üyesi Yahya Güven}

Afyonkarahisar Sağlık Bilimleri Üniversitesi, Diş Hekimliği Fakültesi, Endodonti A.D., Afyonkarahisar

Orcid ID: 0000-0001-7895-8241

\section{Dr. Öğr. Üyesi Mehmet Ünal}

Afyonkarahisar Sağlık Bilimleri Üniversitesi, Diş Hekimliği Fakültesi, Pedodonti A.D., Afyonkarahisar Orcid ID: 0000-0001-9111-6962

Geliş tarihi: 2 Ekim 2020

Kabul tarihi: 11 Mart 2021

doi: 10.5505/yeditepe.2021.70894

\section{Yazışma adresi:}

Ahmet Demirhan Uygun

Afyonkarahisar Sağlık Bilimleri Üniversitesi, Diş

Hekimliği Fakültesi, Endodonti A.D. Afyonkarahisar, 03030, Türkiye

Tel: +90505 7513705

Fax: +902722167080

E-posta: ademirhan100@hotmail.com
ÖZET

Amaç: Bu çalışmanın amacı, $60^{\circ}$ ve $90^{\circ}$ eğimli kanallarda 25/.04 uç boyut ve taper açısına sahip K3XF, 2Shape ve VDW. ROTATE NiTi döner aletlerinin döngüsel yorgunluğa bağlı kırılma dirençlerinin karşılaştıııımasıdır.

Gereç ve Yöntem: Bu çalışmada toplam 90 olmak üzere 30 adet K3XF, 30 adet 2Shape ve 30 adet VDW.ROTATE eğesi kullanıldı. Eğeler iki alt gruba ayrıldıktan sonra $60^{\circ}$ ve $90^{\circ}$ eğimli ve $3 \mathrm{~mm}$ eğim yarıçapına sahip yapay paslanmaz çelik kanallarda döngüsel yorgunluk direnç testine tabi tutuldu. Eğelerin kırılma zamanları bir dijital kronometre yardımıyla belirlendi. Kırık parçaların uzunlukları ise hassas kumpas yardımıyla ölçüldü. Veriler istatistiksel olarak Kruskal Wallis H-testi ve post hoc Tamhane $\mathrm{T} 2$ testi ile istatistiksel olarak incelendi $(\mathrm{p}<0.05)$.

Bulgular: VDW.ROTATE grubu $60^{\circ}$ ve $90^{\circ}$ eğimli her iki kanalda istatistiksel olarak anlamlı şekilde yüksek döngüsel yorgunluk direncine sahipti $(p<0.05)$. K3XF grubu her iki farklı kanalda istatistiksel olarak 2Shape TS1 grubuna göre döngüsel yorgunluğa karşı daha dirençli bulundu $(p<0.05)$. Her iki farklı kanalda eğeler arasında kırık parça uzunlukları kıyaslandığında istatistiksel açıdan fark yoktu ( $p>0.05$ ).

Sonuç: Döngüsel yorgunluğa karşı direncin incelendiği bu çalışmada \%4 taper açısına sahip eğeler kullanılmıştır. VDW. ROTATE eğeleri döngüsel yorgunluğa karşı en yüksek kırııma direncine sahipti. 2Shape eğeleri döngüsel yorgunluğa karşı en düşük dirençli gruptu.

Anahtar kelimeler: Döngüsel yorgunluk, kırılma direnci, Nikel-titanyum, VDW.ROTATE

\section{SUMMARY}

Aim: The aim of this study is to compare the fracture resistance due to cyclic fatigue of K3XF, 2Shape and VDW.ROTATE $\mathrm{NiTi}$ rotary instruments with 25/.04 tip size and taper in artificial stainless steel canals with $60^{\circ}$ and $90^{\circ}$ curvature.

Materials and methods: In this study, a total of $90,30 \mathrm{~K} 3 \mathrm{XF}$, 302 Shape and 30 VDW.ROTATE files were used. After the files were divided into two subgroups, they were subjected to cyclic fatigue resistance tests on artificial stainless steel channels with $60^{\circ}$ and $90^{\circ}$ angle of curvature and $3 \mathrm{~mm}$ radius of curvature. The time to fracture of the files were determined with the help of a digital stopwatch. The lengths of the fractured fragments were measured with a precision caliper. The data were analyzed statistically using Kruskal Wallis $\mathrm{H}$-test and post hoc Tamhane T2 test $(\mathrm{p}<0.05)$.

Results: The VDW.ROTATE group had statistically significant higher cyclic fatigue resistance in both canals with $60^{\circ}$ and $90^{\circ}$ angle of curvature $(p<0.05)$. The K3XF group was statistically more resistant to cyclic fatigue than the 2Shape TS1 group in both canals $(p<0.05)$. There was no statistical significant difference between the lengths of the fractured fragments between the files in both different canals $(p>0.05)$.

Conclusion: In this study, in which resistance to cyclic fatigue 
was examined, files with $4 \%$ taper were used. The VDW. ROTATE files had the highest fracture resistance against cyclic fatigue. The group with the lowest resistance to cyclical fatigue was 2Shape files.

Keywords: Cyclic fatigue, fracture resistance, Nickel-titanium, VDW.ROTATE

\section{GiRiş}

Kök kanal tedavisi sırasında gerçekleşen Nikel-Titanyum (NiTi) döner aletlerin kırılması genellikle klinisyenler tarafından telafi edilemeyen can sıkıcı bir komplikasyondur. Literatüre göre, kök kanal tedavisinde kullanılan aletin kIrılması iki farklı mekanizma ile gerçekleşmektedir; biri torsiyonel (burulma) diğeri de döngüsel (eğilme) yorulmadır. Kanal aletinin uç kısmının sıkışması ancak geri kalan gövde kısmının dönmeye devam etmesi neticesinde gerçekleşen kırılma tipi torsiyonel olarak adlandırılır. Döngüsel yorgunluk ise belirli aralıklarla tekrarlanan sıkışma ve gerilme kuvvetlerine maruz kalma sonucunda gerçekleşir. Kök kanal tedavisinde döngüsel yorgunluktan dolayı oluşan alet kırılmasının daha sık karşılaşıldığı iddia edilmektedir. ${ }^{1,2}$

$\mathrm{NiTi}$ döner aletlerin döngüsel yorgunluk dirençlerinin klyaslandığı çalışmalarda eğimli kök kanallarında kök kanal aletinin taper açısının artmasıyla döngüsel yorgunluk direncinin azaldığı ve alet kırılması riskinin artığı gösterilmiştir. ${ }^{3,4}$ Ayrıca yüksek taper açısına sahip aletlerin kök kanal duvarlarında daha çok stres oluşumuna yol açtığı, köklerin kırılma direncini azalttığı ve vertikal kök kırığı oluşma riskini artırdığı gösterilmiştir.5, 6 Kök kanal tedavisinde kullanılan ticari NiTi döner aletler farklı taper açılarında üretilirler ancak genellikle bu sistemlerin final bitim eğeleri \%6 civarında bir açıya sahiptir. Dolayısıyla döngüsel yorgunluk direnç testi çalışmalarında kullanılan kanal aletleri de ağırlıklı olarak \%6 civarında bir taper açısına tablo 1 sahiptir. Literatürde azaltılmış taper açısına sahip döner aletlerin döngüsel yorgunluk direnci ile ilgili daha az çalışma bulunmaktadır.?

Tablo 1. K3XF, 2 Shape ve VDW ROTATE eğerlerinin 2 farklı açıdaki kanallarda kırılma zamanları ( $\mathrm{sn}$ ) ve kırık parça uzunlukları $(\mathrm{mm})$ ortalama ve standart sap ma değerler

\begin{tabular}{|c|c|c|c|c|}
\hline & \multicolumn{2}{|c|}{$60^{\circ}$} & \multicolumn{2}{|c|}{$90^{\circ}$} \\
\hline & $\begin{array}{l}\text { Kirilma zamanı } \\
\text { (sn) }\end{array}$ & $\begin{array}{l}\text { Kırık parça } \\
\text { uzunluğu } \\
(\mathrm{mm})\end{array}$ & $\begin{array}{l}\text { Kirilma zamanı } \\
\text { (sn) }\end{array}$ & $\begin{array}{l}\text { Kırık parça } \\
\text { uzunluğu } \\
(\mathrm{mm})\end{array}$ \\
\hline K3XF 25/.04 & $123 \pm 17^{b}$ & $4,98 \pm 0,32^{2}$ & $102 \pm 14^{b}$ & $5,15 \pm 0,33^{2}$ \\
\hline 2Shape TS1 & $82 \pm 9^{c}$ & $4,94 \pm 0,22^{2}$ & $37 \pm 7^{6}$ & $5,1 \pm 0,23^{\circ}$ \\
\hline VDW.ROTATE 25/.04 & $194 \pm 42^{\mathrm{a}}$ & $5,06 \pm 0,3^{\circ}$ & $123 \pm 13^{\circ}$ & $5,21 \pm 0,28^{3}$ \\
\hline
\end{tabular}

Aynı sütundaki farklı üst simge harfler istatistiksel olarak anlamlı farklılık olduğu anlamına gelir $(\mathrm{P}<0.05)$

K3XF sistemi (Orange, Kaliforniya, $A B D$ ) daha önceki yıllarda üretilen $\mathrm{K} 3$ (Orange, Kaliforniya, $\mathrm{ABD}$ ) ve Twisted File ( $T F$, Orange, Kaliforniya, ABD) sistemlerine benzer olarak üretilmiştir. TF gibi R-faz Isıl işlem teknolojisine sahiptir ancak bükülme yerine K3 gibi taşlama yöntemi ile üretilmiştir. K3XF sistemi farklı konisite ve uç boyutlarına sahip birçok eğe içermektedir.8, 9 2Shape (Micro-Mega, Besançon, Fransa) sistemi T.wire ısıl işlem teknolojisi ile üretilen 2 adet eğeden oluşan NiTi döner sistemidir. TS1 ve TS2 sırasıyla 25/.04 ve 25/.06 uç boyut ve taper açısına sahiptir. ${ }^{10,11}$ VDW.ROTATE ( VDW, Munich, Almanya) son yıllarda ısıl işlem teknolojisi ile üretilen ve birçok eğeden oluşan bir sistemdir. Sistemin eğeleri normal ve dar kanallarda kullanılmak üzere 0,06 ve 0,04 taper açısına sahip eğelerden oluşmaktadır. Literatürde bu sistemlerin döngüsel yorgunluk dirençlerinin kıyaslandığı az sayıda çaışma vardır. ${ }^{12,13}$ Bu çalışmanın amacı, 0,25 mm apikal uç boyuta ve \%4 taper açısına sahip yeni döner alet sistemlerinin döngüsel yorgunluk dirençlerini kıyaslamaktır. Çaış̧manın sıfır hipotezi, K3XF (25/.04), 2Shape TS1 ve VDW. ROTATE (25/.04) NiTi döner aletlerinin döngüsel yorgunluk dirençleri bakımından istatistiksel olarak anlamlı fark olmayacağıdır.

\section{GEREÇ VE YÖNTEM}

Bu çalışmada 30 adet K3XF (25-0.04), 30 adet 2Shape TS1 ve 30 adet VDW.ROTATE (25-0.04) kullanıldı. Deneyden önce tüm örnekler stereomikroskop altında üretim hatası yönünden incelendi ve kusurlu bir eğe ile karşılaşılmadı.

Döngüsel yorulma testi için 3 boyutlu olarak farklı yönlerde ayarlanabilen bir test düzeneği oluşturuldu (Şekil 1).

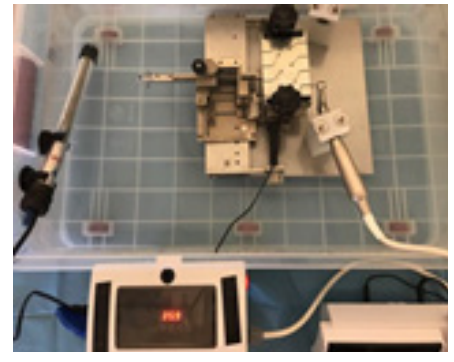

Şekil 1. Döngüsel yorgunluk direnci test düzeneği

Örnekler 2 alt gruba ayrıldı ( $n=15), 60^{\circ}$ ve $90^{\circ}$ eğimli ve 3 $\mathrm{mm}$ eğim yarıçapına sahip yapay paslanmaz çelik kanallar döngüsel yorgunluk direnç testi için tercih edildi. ${ }^{14}$ Döner aletler uç kısmı kanal kurvatürünü 5 mm geçecek şekilde yerleştirildi. Kırılmanın takibi ve kırılacak parçanın kaybını engellemek için yapay kanalların üstü bir cam blok ile örtüldü. Üretici talimatlarına uygun olarak 350 rpm hızda VDW Gold Reciproc endodontik motor kullanılarak test gerçekleştirildi. Döngüsel yorgunluk direnç testi $35^{\circ} \mathrm{C}( \pm 2)$ sıcaklıkta distile su içinde gerçekleştirildi. Dijital bir kronometre ile kırılma zamanları ölçüldü. Kırık parça uzunlukları hassas bir kumpas yardımı ile ölçüldü ve eğelerin kırık yüzeyleri taramalı elektron mikroskobu (LEO 1430 VP, Zeiss Oberkochen, Almanya) altında incelendi (Şekil 2-4). 


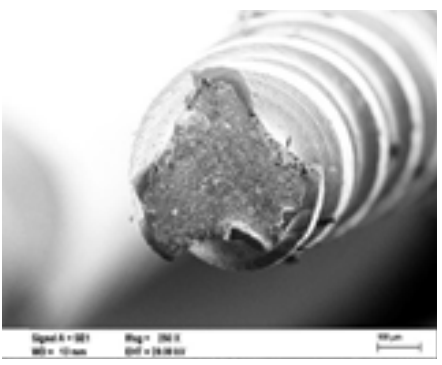

Şekil 2. K3XF eğesinin kırık yüzeyinin taramalı elektron mikroskobu görüntüsü

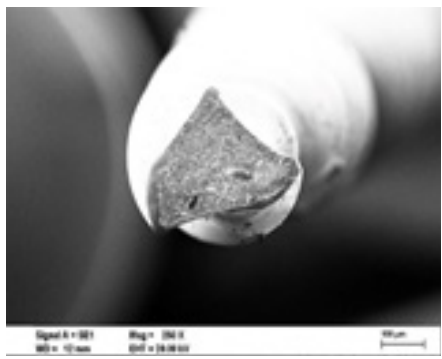

Şekil 3. 2Shape eğesinin kırık yüzeyinin taramalı elektron mikroskobu görüntüsü

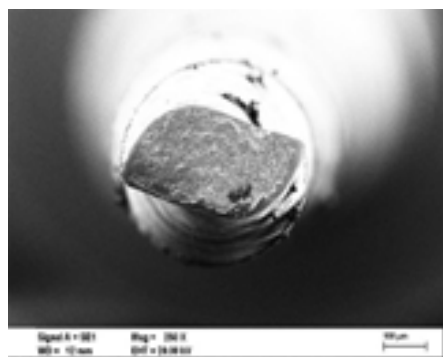

Şekil 4.VDW.ROTATE eğesinin kırık yüzeyinin taramalı elektron mikroskobu görüntüsü

Eğelerin kırılma zamanı verileri istatistiksel olarak Kruskal Wallis H-testi ve post hoc Tamhane T2 testi ile istatistiksel olarak incelendi. Sonuçlar istatistiksel olarak \%5 anlamlılık oranında değerlendirildi (SPSS v23.0; IBM Corp, Armonk, NY, ABD).

\section{BULGULAR}

Eğelerin $60^{\circ}$ ve $90^{\circ}$ eğimli kanallarda kırılma zamanları (sn) ve kırık parça uzunlukları (mm) ortalama ve standart sapma verileri' de verilmiştir. Her iki eğimli kanalda VDW. ROTATE grubu istatistiksel olarak anlamlı şekilde yüksek döngüsel yorgunluk direncine sahipti $(p<0.05)$. K3XF grubu her iki farklı kanalda da istatistiksel olarak 2Shape TS1 grubuna göre döngüsel yorgunluğa karşı daha dirençli bulundu $(p<0.05)$. Her iki farklı kanalda eğeler arasında kırık parça uzunlukları kıyaslandığında istatistiksel açıdan fark yoktu ( $p>0.05)$.

\section{TARTIŞMA}

Endodontik tedavinin başarısı birçok faktöre bağlıdır. Bu faktörlerden mekanik olarak kök kanalının genişletilmesi çok önem arz etmektedir. Ancak literatürde kök kanalında hangi boyuta kadar genişletme yapılacağı tartışma konusudur. Kök kanal genişletmesinin arttıkça apikal iyileşmenin azaldığı rapor edilmiştir. ${ }^{15,16}$ Saini ve ark. ${ }^{17}$ ise yaptıkları bir çalışmada, kök kanalında ilk sıkışan eğenin 3 üstüne kadar yapılan apikal genişletmenin ye- terli olduğu ve daha fazla yapılan genişletmenin periapikal iyileşmeye bir katkısının olmadığını göstermişlerdir. Ayrıca kök kanal genişletmesinin vertikal kırılma direncini düşürdüğü yapılan çalışmalar neticesinde bilinmektedir. ${ }^{18-20}$ Dolayısıyla yüksek taper açısına sahip döner aletlerden kaçınmak ve orijinal kanal kurvatürünü korumak gibi minimal invaziv yaklaşımlar daha popüler hale gelmektedir. Bunlara ilaveten daha az taper açısına sahip döner aletlerin kullanımıyla döngüsel yorgunluk direnci artırılıp kök kanalında alet kırılması riski azaltılmaktadır. Biz de çalışmamızda daha az taper açısına sahip NiTi döner aletlerin 2 farklı açıya sahip paslanmaz çelik kanallarda döngüsel yorgunluk dirençlerini inceledik. VDW. ROTATE eğeleriyle diğer eğeler arasında her iki kanalda da istatistiksel olarak anlamlı fark vardı $(p<0.05)$. 2Shape TS1 eğeleri de K3XF eğelerinden istatistiksel olarak daha yüksek kırılma direncine sahipti $(p<0.05)$. Böylece bu çalışmanın sıfır hipotezi reddedilmiştir.

Literatürde döngüsel yorgunluğu ölçmek için birbirinden farklı birçok düzenek kullanılmıştır. Bunlar başlıca sabit (statik) ve hareketli (dinamik) olmak üzere ikiye ayrılabilir. Hareketli düzenekler sabite ek olarak aksiyel hareketi de içermektedir ${ }^{21}$ Aksiyel hareket ile NiTi döner aletlerinde kanal kurvatüründe oluşan stresin tek bir noktada toplanmak yerine belli bir bölgeye dağıldığı ve kırılma direncinin arttığı gösterilmiştir. ${ }^{22}$ Bizim çalışmamızda standardizasyonun daha iyi sağlanabilmesi açısından sabit bir model içinde eğeler döngüsel yorgunluk direnç testine tabi tutulmuştur. Castello-Escriva ve ark. ${ }^{23}$ eğim yarıçapı açısının eğelerin döngüsel yorulma direncini etkileyen önemli faktörler olduğunu göstermiştir. Literatürde \%6 taper açısına sahip eğeler genellikle $60^{\circ}$ eğim açısına sahip yapay kanallarda teste tabi tutulmaktadırlar, ancak daha düşük taper açısına sahip eğeler $60^{\circ}$ eğime sahip kanallara ilaveten $90^{\circ}$ eğimli veya S harfi şeklinde çift eğimli yapay kanallarda döngüsel yorgunluk direnç testine tabi tutulmuşlardır. ${ }^{72}$ Biz de \%4 taper açısına sahip eğeleri incelediğimiz çalışmamızda, döner aletin üç boyutlu olarak konumlandırılabildiği sabit bir test düzeneği içerisinde yarıçapı $3 \mathrm{~mm}$ olan $60^{\circ}$ ve $90^{\circ}$ eğime sahip yapay paslanmaz çelik kanallarda döngüsel yorgunluk direnç testi gerçekleştirilmiştir. ${ }^{14,25-27}$ Önceki çalışmalarla uyumlu olarak 90 eğime sahip kanalların eğelerin kırılma dirençlerini daha hızlı bir şekilde azalttığı görülmüştür. 4, 27 Son yıllarda ortam koşullarının döngüsel yorgunluk direnç testlerini etkilediği iddia edilmiştir. ${ }^{28-30}$ Daha önceden yapılan çalışmalarda sıcaklığın eğelerin fiziksel özelliklerini etkilediği ve kanal içi sıcaklıkta bu çalışmaların yapılmasının daha doğru olacağı belirtilmiştir. ${ }^{28,31}$ Literatürde yapılan az sayıdaki çalışmanın sonuçlarına göre, kanal içi sıcaklığın vücut sıcaklığından bir miktar daha düşük olarak $31^{\circ} \mathrm{C}$ ve $35^{\circ} \mathrm{C}$ aralığında olduğu belirtilmiştir. ${ }^{32,33} \mathrm{Bu}$ nedenden dolayı döngüsel yorgunluk 
direnç testi bir termostat yardımı ile kontrol edilen $35^{\circ} \mathrm{C}$ ( \pm 2$)$ sıcaklığındaki su içerisinde gerçekleştirildi.

Eğelerin mekanik özelliklerini dolayısıyla kırılma direncini etkileyen faktörlerden biri eğenin geometrik tasarımıdır. Geometrik tasarım enine kesit ile ilişkilidir ve kırılma direncini etkilediği daha önce yapılan çalışmalar ile gösterilmiştir. ${ }^{34,35} \mathrm{~S}$ şeklinde enine kesite sahip eğelerin dörtgen veya üçgen şekilli enine kesitli eğelere nazaran daha yüksek kırılma direncine sahip oldukları gösterilmiştir. ${ }^{36,37}$ VDW.ROTATE eğeleri de S şeklinde enine kesite, 2Shape TS1 dışbükey üçgen ve K3XF içbükey üçgen benzeri farklı bir enine kesite sahiptir. Bu çalışmadaki döngüsel yorgunluk direncindeki fark enine kesit tasarımlarından kaynaklanmış olabilir. Eğelerin kırılma direncini etkileyen tek faktör sadece geometrik tasarımları değildir. Aynı zamanda üretildikleri ısı işlem teknolojileri de alaşımların kırılma direncini etkilemektedir. ${ }^{26,38,39}$ Firmalar tarafından ürettikleri eğelerin sahip oldukları alaşım ve üretim süreçleri tam olarak açıklanmamakta ve farklı isimlerle adlandırılmaktadır. 2Shape T.wire ve K3XF ise R-phase olarak adlandırılan alaşımlara sahiptir.9,10 VDW. ROTATE eğesi bu eğelerden daha sonraki yıllar içerisinde üretilen yeni bir eğedir ve üreticisi tarafından alaşımına dair paylaşılan bir bilgi yoktur. Keskin ve ark. ${ }^{12}$ yaptıkları bir diferansiyel taramalı kalorimetri çalışmasında, VDW. ROTATE, Reciproc Blue ve Reciproc gibi Isıl işlem görmüş eğelerin Östenit bitiş (Af) sıcaklıklarını sırasıyla $33^{\circ} \mathrm{C}, 35^{\circ} \mathrm{C}$ ve $41^{\circ} \mathrm{C}$ bulmuşlardır. Aynı çalışmada ise geleneksel bir $\mathrm{NiTi}$ alaşıma sahip olan Mtwo eğesi $15^{\circ} \mathrm{C}$ Af sıcaklığına sahipti. Bu bulgular VDW.ROTATE eğelerinin geleneksel bir NiTi eğeden daha yüksek kırılma direncine sahip olduğunu açıklamaktadır.

Literatürdeki VDW.ROTATE eğeleri ile ilgili az sayıda çalışma bulunmaktadır. Gündoğar ve ark. ${ }^{11} 2020$ yılında yaptıkları çalışmasında VDW.ROTATE eğesinin döngüsel yorgunluk direncini diğer bütün eğelerden daha yüksek bulmuşlardır. Uslu ve ark. ${ }^{40} \mathrm{~S}$ şeklinde çift kurvatürlü yapay bir kanala sahip düzenekte yaptıkları çalışmalarında, VDW.ROTATE eğeleri ve HYFlex CM eğeleri arasında istatistiksel bir fark bulamazken, 2Shape eğelerinin daha düşük döngüsel yorgunluk direncine sahip olduklarını göstermişlerdir. Bizim çalışmamızın sonuçları da bu iki çalışma ile uyum içerisindedir. Bilgimiz dahilinde literatürde 2Shape ve K3XF eğelerinin döngüsel yorgunluk direncini kıyaslayan bir çalışma bulunmamaktadır. Daha yeni bir teknoloji ile üretilmiş olmasına rağmen 2Shape TS1 eğeleri K3XF eğelerinden düşük döngüsel yorulma direncine sahipti.

\section{SONUÇLAR}

Çalışmanın sınırları dahilinde, \%4 taper açısına sahip farklı tasarımlara ve ısıl işlem teknolojilerine sahip NiTi döner aletlerin döngüsel yorgunluk dirençleri kıyaslandı. VDW.ROTATE eğeleri istatistiksel olarak en yüksek kırıl- ma direncine sahipti. 2Shape TS1 eğesi ile K3XF arasında istatistiksel olarak anlamlı fark vardı. Eğelerin kırılmasını etkileyen tek faktör sadece döngüsel yorgunluk direnci değildir, bu nedenle söz konusu eğelerin klinik kullanımlarında dikkatli olmakta fayda vardır ve bu eğelerle ilgili yapılacak klinik çalışmalara intiyaç vardır.

\section{Teşekkür}

Bu çalışma, Afyonkarahisar Sağlık Bilimleri Üniversitesi Bilimsel Araştırma Projeleri Komisyonu tarafından 19.Diş.001 numaralı proje ile desteklenmiştir.

\section{KAYNAKLAR}

1. Cheung G, Peng B, Bian Z, Shen Y, Darvell B. Defects in ProTaper S1 instruments after clinical use: fractographic examination. Int Endod J 2005;38:802-809.

2. Shen Y, Haapasalo M, Cheung GS-p, Peng B. Defects in nickel-titanium instruments after clinical use. Part 1: Relationship between observed imperfections and factors leading to such defects in a cohort study. J Endod 2009;35:129-132.

3. Pérez-Higueras JJ, Arias A, José C, Peters OA. Differences in cyclic fatigue resistance between ProTaper Next and ProTaper Universal instruments at different levels. J Endod 2014;40:1477-1481.

4. Adıgüzel M, Capar ID. Comparison of cyclic fatigue resistance of WaveOne and WaveOne Gold small, primary, and large instruments. J Endod 2017;43:623-627.

5. Zandbiglari T, Davids $H$, Schäfer E. Influence of instrument taper on the resistance to fracture of endodontically treated roots. Oral Surg Oral Med Oral Pathol Oral Radiol Endod 2006;101:126-131.

6. Versluis $A$, Messer $H$, Pintado M. Changes in compaction stress distributions in roots resulting from canal preparation. Int Endod J 2006;39:931-939.

7. Hülsmann M, Donnermeyer D, Schäfer E. A critical appraisal of studies on cyclic fatigue resistance of engine-driven endodontic instruments. Int Endod J 2019;52:1427-1445.

8. Pérez-Higueras JJ, Arias A, José C. Cyclic fatigue resistance of K3, K3XF, and twisted file nickel-titanium files under continuous rotation or reciprocating motion. J Endod 2013;39:1585-1588.

9. Shen Y, Zhou H-m, Wang Z, Campbell L, Zheng Y-f, Haapasalo M. Phase transformation behavior and mechanical properties of thermomechanically treated K3XF nickel-titanium instruments. J Endod 2013;39:919-923.

10. 1Elnaghy AM, Elsaka SE. Cyclic fatigue resistance of one curve, 2Shape, ProFile vortex, vortex blue, and RaCe nickel-titanium rotary instruments in single and double curvature canals. J Endod 2018;44:1725-1730.

11. Gündoğar M, Uslu G, Özyürek T, Plotino G. Comparison of the cyclic fatigue resistance of VDW. ROTATE, TruNatomy, 2Shape, and HyFlex CM nickel-titanium rotary files at body temperature. Restor Dent Endod 2020;45. 
12. Keskin C, Yilmaz ÖS, Keleş A, Inan U. Comparison of cyclic fatigue resistance of Rotate instrument with reciprocating and continuous rotary nickel-titanium instruments at body temperature in relation to their transformation temperatures. Clin Oral Investig 2020;1-7.

13. 1Uygun AD. Cyclic fatigue resistance of VDW. ROTATE and Reciproc Blue nickel-titanium files at root canal temperature. J Dent Res 2020;14:2.

14. Pruett JP, Clement DJ, Carnes Jr DL. Cyclic fatigue testing of nickel-titanium endodontic instruments. J Endod 1997;23:77-85.

15. Strindberg LZ. The dependence of the results of pulp therapy on certain factors-an analytical study based on radiographic and clinical follow-up examination. Acta Odontol Scand 1956;14:1-175.

16. Hoskinson SE, Ng Y-L, Hoskinson AE, Moles DR, Gulabivala $\mathrm{K}$. A retrospective comparison of outcome of root canal treatment using two different protocols. Oral Surg Oral Med Oral Pathol Oral Radiol Endod 2002;93:705715.

17. Saini HR, Tewari S, Sangwan P, Duhan J, Gupta A. Effect of different apical preparation sizes on outcome of primary endodontic treatment: a randomized controlled trial. J Endod 2012;38:1309-1315.

18. Bergmans L, Van Cleynenbreugel J, Wevers M, Lambrechts P. Mechanical root canal preparation with NiTi rotary instruments: rationale, performance and safety. Am J Dent 2001;14:324-333.

19. Cohen S, Berman LH, Blanco L, Bakland L, Kim JS. A demographic analysis of vertical root fractures. $J$ Endod 2006;32:1160-1163.

20. Topçuoğlu HS, Arslan H, Keleş A, Köseoğlu M. Fracture resistance of roots filled with three different obturation techniques. Medicina oral, patologia oral y cirugia bucal 2012;17:e528.

21. Dederich DN, Zakariasen KL. The effects of cyclical axial motion on rotary endodontic instrument fatigue. Oral Surg Oral Med Oral Pathol Oral Radiol Endod 1986;61:192-196.

22. Li U-M, Lee B-S, Shih C-T, Lan W-H, Lin C-P. Cyclic fatigue of endodontic nickel titanium rotary instruments: static and dynamic tests. J Endod 2002;28:448-451.

23. Castelló-Escrivá R, Alegre-Domingo $T$, Faus-Matoses V, Román-Richon S, Faus-Llácer VJ. In vitro comparison of cyclic fatigue resistance of ProTaper, WaveOne, and Twisted Files. J Endod 2012;38:1521-1524.

24. Ferreira F, Adeodato C, Barbosa I, Aboud L, Scelza P, Zaccaro Scelza M. Movement kinematics and cyclic fatigue of NiTi rotary instruments: a systematic review. Int Endod J 2017;50:143-152.

25. Capar ID, Kaval ME, Ertas H, Sen BH. Comparison of the cyclic fatigue resistance of 5 different rotary pathfinding instruments made of conventional nickel-titani- um wire, M-wire, and controlled memory wire. J Endod 2015;41:535-538.

26. Uygun A, Kol E, Topcu M, Seckin F, Ersoy I, Tanriver $M$. Variations in cyclic fatigue resistance among ProTaper Gold, ProTaper Next and ProTaper Universal instruments at different levels. Int Endod J 2016;49:494-499.

27. Özyürek T, Gündoğar M, Uslu G, Yılmaz K, Staffoli S, Grande N, Plotino G, Polimeni A. Cyclic fatigue resistances of Hyflex EDM, WaveOne gold, Reciproc blue and 2shape NiTi rotary files in different artificial canals. Odontology 2018; 106:408-413.

28. de Vasconcelos RA, Murphy $S$, Carvalho CAT, Govindjee RG, Govindjee S, Peters OA. Evidence for reduced fatigue resistance of contemporary rotary instruments exposed to body temperature. J Endod 2016;42:782-787.

29. Inaghy A, Elsaka S. Effect of sodium hypochlorite and saline on cyclic fatigue resistance of WaveOne Gold and Reciproc reciprocating instruments. Int Endod $\mathrm{J}$ 2017;50:991-998.

30. Keles A, Ozyurek EU, Uyanik MO, Nagas E. Effect of temperature of sodium hypochlorite on cyclic fatigue resistance of heat-treated reciprocating files. J Endod 2019;45:205-208.

31. Plotino G, Grande NM, Bellido MM, Testarelli L, Gambarini G. Influence of temperature on cyclic fatigue resistance of ProTaper Gold and ProTaper Universal rotary files. J Endod 2017;43:200-202.

32. de Hemptinne $F$, Slaus $G$, Vandendael $M$, Jacquet $W$, De Moor RJ, Bottenberg P. In vivo intracanal temperature evolution during endodontic treatment after the injection of room temperature or preheated sodium hypochlorite. J Endod 2015;41:1112-1115.

33. Cunningham WT, Balekjian AY. Effect of temperature on collagen-dissolving ability of sodium hypochlorite endodontic irrigant. Oral Surg Oral Med Oral Pathol Oral Radiol Endod 1980;49:175-177.

34. Xu X, Eng M, Zheng Y, Eng D. Comparative study of torsional and bending properties for six models of nickel-titanium root canal instruments with different cross-sections. J Endod 2006;32:372-375.

35. Grande N, Plotino G, Pecci R, Bedini R, Malagnino V, Somma F. Cyclic fatigue resistance and three-dimensional analysis of instruments from two nickel-titanium rotary systems. Int Endod J 2006;39:755-763.

36. Plotino G, Grande N, Testarelli L, Gambarini G. Cyclic fatigue of Reciproc and WaveOne reciprocating instruments. Int Endod J 2012;45:614-618.

37. Dagna A, Poggio C, Beltrami R, Colombo M, Chiesa M, Bianchi S. Cyclic fatigue resistance of OneShape, Reciproc, and WaveOne: An in vitro comparative study. J Conserv Dent 2014;17:250.

38. Ha J-H, Kim SK, Cohenca N, Kim H-C. Effect of R-phase heat treatment on torsional resistance and cyclic fatigue 
fracture. J Endod 2013;39:389-393.

39. Gündoğar M, Özyürek T. Cyclic fatigue resistance of OneShape, HyFlex EDM, WaveOne Gold, and Reciproc Blue nickel-titanium instruments. J Endod 2017;43:11921196.

40. Uslu G, Gundogar M, Özyurek T, Plotino G. Cyclic fatigue resistance of reduced-taper nickel-titanium (NiTi) instruments in doubled-curved (S-shaped) canals at body temperature. J Dent Res 2020;14: 2. 